\title{
7 Geistiges Eigentum
}

Ausgangsthese für dieses Kapitel: Die Anwendung des grundgesetzlich garantierten allgemeinen Eigentumsrechts (entsprechend GG Art. 14 Abs. 1) auf immaterielle Objekte, sprich: die weitgehende Übertragung von „Sach-Eigentum“ auf „geistiges Eigentum“ gehört zu den rechtswissenschaftlichen und -politischen Fehleinschätzungen, die dazu beigetragen haben, dass das Urheberrecht für Bildung und Wissenschaft eher behindernd als befördernd wirkt. In 7.1 wird auf die weitgehenden Unterschiede von Eigentum und geistiges Eigentum hingewiesen und damit die Berechtigung, die Regulierung von Eigentum auf geistiges Eigentum zu übertragen. Darüber hinaus wird bezweifelt, ob geistiges Eigentum im Wissenschaftsurheberrecht überhaupt Sinn ergibt. In 7.2 wird die vermögensrechtliche Begründung des geistigen Eigentums über Entscheidungen des BVerfG dargestellt und für Wissenschaft in Frage gestellt. In 7.3 wird dargestellt, wie in der Systematik des Urheberrechts geistiges Eigentum aus der Fiktion des individuellen Schöpfers abgeleitet wird. Dies ist das zentrale Beispiel für das Als-ob-Verhalten des Urheberrechts, aus dem sich weitere Restriktionen für die Nutzung des publizierten Wissens ableiten. In 7.4 werden die verschiedenen Interessen an dem Konzept des geistigen Eigentums analysiert - mit dem Zwischenergebnis, dass geistiges Eigentum weitgehend eine folgenreiche ideologische Funktion hat. Der Schutz von geistigem Eigentum ist zunehmend dysfunktional geworden. In 7.5 wird geistiges Eigentum in den Kontext anderer Grundrechte wie Wissenschafts- oder Informationsfreiheit gestellt - mit dem Ergebnis, dass Einschränkungen in geistiges Eigentum eher gerechtfertigt sind als Einschränkungen von Wissenschaftsfreiheit. In 7.6 wird die Kritik am Konzept des geistigen Eigentums noch einmal zusammengefasst. Die Schlussthese in 7.7 besagt, dass das Beharren auf Eigentumsansprüchen zu den Als-ob-Phänomenen gehört, zu den Fiktionen, die in der Gegenwart nicht mehr zu halten sind - weder aus der Sicht und den Interessen der Akteure in Bildung und Wissenschaft und erst recht nicht aus dem Interesse der Öffentlichkeit, Wissen freizügig nutzen zu können.

\subsection{Vom „Eigentum“ im Grundgesetz zum „Geistigen Eigentum“ im Urheberrecht}

„Das Urheberrecht ist das Nutzungsrecht ‘Eigentum’ im Sinne des Art. 14 Abs. 1“ so stellt es das BVerfG im ersten Satz der Schulprivileg-Entscheidung ${ }^{211}$ fest. Die Eigentumsgarantie des GG wird als verfassungsrechtliche Grundlage für die Rechte

211 BVerfGE 31, 229 - Schulbuchprivileg.

Ә Open Access. (C) 2020 Rainer Kuhlen (c))BY This work is licensed under a Creative Commons Attribution 4.0 License. https://doi.org/10.1515/9783110693447-007 
am Eigentum und dessen Schutz gesehen. „Geistiges Eigentum“ kommt als Benennung im GG nicht vor - erklärbar mit dem Zeitpunkt der Entstehung des GG, denn 1948 war so etwas wie geistiges Eigentum den „Vätern“ des Grundgesetzes nicht bewusst - auch kaum das Konzept des Immaterialguts, welches im 19. Jahrhundert „geistiges Eigentum“ durch das Recht regulierbar gemacht hat. Eigentum war für das GG Sacheigentum. Das wird an dem folgenden Zitat von Carlo Schmid deutlich 1948 im Ausschuss für Grundsatzfragen des Parlamentarischen Rates vorgetragen.

Die Formulierung den Gedanken zum Ausdruck bringen, es gebe keine aus der Natur fließende Definition des Inhalts des Eigentums, und das Eigentum, nämlich konkret das Ausmaß, in dem ein Individuum über Sachen verfügen könne, und was es bedeute, ein eigentümliches Recht an einer Sache zu haben, sei notwendig vom Gesetzgeber her zu bestimmen. ${ }^{212}$

Ebenfalls 1948 wurde die (völkerrechtlich nicht-verbindliche, aber mit hohem moralischen Anspruch verbundene) Allgemeine Erklärung der Menschenrechte (AEMR) beschlossen. In ihr wird Eigentum als Grundrecht bzw. Menschenrecht angesehen: ${ }^{213}$ „Jeder hat das Recht, sowohl allein als auch in Gemeinschaft mit anderen Eigentum innezuhaben“ und: „Niemand darf willkürlich seines Eigentums beraubt werden.“ „Geistiges Eigentum“ wird zwar nicht erwähnt, aber ist, wie an dem folgenden Zitat deutlich wird, doch wohl mitgedacht: ,Jeder Mensch hat das Recht auf Schutz der moralischen und materiellen Interessen, die sich aus jeder wissenschaftlichen, literarischen oder künstlerischen Produktion ergeben, deren Urheber er ist." Hiermit ist nicht nur die intellektuelle Produktion, sondern in der Verbindung von moralischen und materiellen Interessen auch die monistische Struktur angesprochen, die für Urheberrechtsregulierungen vor allem im kontinentaleuropäischen Bereich bestimmend ist (vgl. 6.5).

Mit „Geistigem Eigentum“ haben sich die beiden völkerrechtlich verbindlichen Internationalen WIPO-Pakte über bürgerliche und politische Rechte vom 16. Dezember 1966 und Pakt über wirtschaftliche, soziale und kulturelle Rechte vom 19. Dezember 1966 schwerer getan. In diesen beiden Texten ist sogar von „Eigentum“ insgesamt nicht die Rede. Offenbar waren damals (und wohl auch noch heute) weltweit die ideologischen Unterschiede über die Reichweite von Eigentum so unterschiedlich, als dass eine völkerrechtlich verbindliche Festlegung von Eigentum

212 Das Zitat ist der Dissertation von (Bajon 2010) Interessenausgleich im Wissenschaftsurheberrecht entnommen; dort mit Verweis auf BVerfG NJW 2001, 1783, 1784.

An diesem Zitat, in dem Schmid Satz 2 von Art. 14 Abs. 1 GG ([,„Inhalt und Schranken werden durch die Gesetze bestimmt“) erläuterte, ist allerdings weniger die terminologische Klärung von „Eigentum“ wichtig, sondern die Feststellung, dass es keine quasi naturrechtliche Bestimmung von Eigentum gebe. Vielmehr obliege es dem Gesetzgeber, in positiven Gesetzen festzulegen, in welchem Umfang Eigentum geschützt werden soll.

213 AEMR, Art. 17 Abs. 1 - Recht auf Eigentum; Art 17 Abs. 2. 
geschweige denn von geistigem Eigentum über diese Pakte möglich gewesen wäre. Die Regelung des geistigen Eigentums wurde anderen internationalen Vereinbarungen überlassen. Allerdings wird auch in der bis heute noch Anwendung findenden internationalen Urheberrechtsregulierung, Berner Übereinkommen zum Schutz von Werken der Literatur und Kunst von 1887 (letzte Fassung 2004), kein direkter Bezug auf ,geistiges Eigentum“ als urheberrechtlich relevanten Begriff genommen. Es finden sich lediglich Verweise auf das Internationale Büro für geistiges Eigentum und dessen 1967 eingerichtete Nachfolgeorganisation, die UN Weltorganisation für geistiges Eigentum (WIPO). Mit dem Titel der WIPO ist aber „geistiges Eigentum“ in der internationalen Nomenklatur verbindlich eingeführt. Der spätestens seit Mitte der 80er Jahre deutlich erkennbaren kommerziellen Bedeutung von immateriellen Produkten (und damit von geistigem Eigentum) wurde dann international ebenfalls im Titel des 1995 in Kraft getretenen Agreement on Trade-Related Aspects of Intellectual Property Rights (TRIPS) als Bestandteil der World Trade Organization (WTO) Rechnung getragen.

Das deutsche Urheberrecht spricht bis heute an keiner Stelle von ,geistigem Eigentum“. Man kann aber davon ausgehen, dass immer dann, wenn im Zusammenhang von urheberrechtlichen Themen in Rechtsetzung, Rechtsprechung und Rechtswissenschaft der Urheberrechtsdiskussion von „Eigentum“ die Rede ist, nicht „Sacheigentum“ gemeint ist, sondern eben das Recht an immateriellen Objekten. „Geistiges Eigentum“ ist omnipräsent und wird in den letzten 50 Jahren nicht mehr in Frage gestellt. Das war zur Zeit der 65-er Reform des UrhG noch anders. In den Ausführungen zu dieser Reform wurde noch einmal (und vielleicht zum letzten Mal in einer Urheberrechts-Reform) deutlich gemacht, dass die Übertragung von „Eigentum“ auf „geistiges Eigentum“ problematisch und in der Konsequenz „unrichtig“ ist. Die zentrale Aussage von 1965, dass „Urhebergut ... seinem Wesen nach Mitteilungsgut" sei, ist in den folgenden Jahren immer mehr zugunsten des vermögensrechtlichen Aspekts des geistigen Eigentums in den Hintergrund gerückt. ${ }^{214}$

Insbesondere [ist] aus Kreisen der Urheber eingewandt worden, das Urheberrecht müsse als sogenanntes geistiges Eigentum dem Sacheigentum gleichgestellt werden und dem Urheber eine unbeschränkte Herrschaft über sein Werk gewähren. Hierbei wird jedoch verkannt, daß zwischen dem Sacheigentum und dem Urheberrecht grundsätzliche Unterschiede bestehen. Sinn des Sacheigentums ist es, dem Eigentümer die alleinige Herrschaft über die ihm gehörende Sache zu geben, damit er andere von der Benutzung ausschließen kann. Urhebergut ist dagegen seinem Wesen nach Mitteilungsgut. Ein Geisteswerk soll gerade - jedenfalls von dem Augenblick an, in dem der Urheber es veröffentlicht hat - in seinem Gedankenoder Gefühlsinhalt möglichst vielen anderen Menschen zugänglich gemacht werden. Im Gegensatz zum Sacheigentum ist das Urheberrecht also letztlich nicht dazu bestimmt, andere von der Benutzung des Werkes auszuschließen. Es soll vielmehr in erster Linie dem Urheber

214 Dazu ausführlicher aus Sicht des BVerfG im nächsten Abschnitt 7.2. 
die rechtliche Grundlage dafür geben, Art und Umfang der Benutzung seines Werkes zu überwachen und aus dessen Verwertung Einnahmen zu erzielen. Die Folgerung, die aus der Gleichstellung des „geistigen Eigentums“ mit dem Sacheigentum gezogen wird, daß nämlich jede sachliche Beschränkung des Urheberrechts - auch im Interesse der Allgemeinheit - mit der Rechtsnatur des Urheberrechts nicht vereinbar sei, ist hiernach nicht berechtigt. Diese Folgerung ist darüber hinaus auch deswegen unrichtig, weil schon das Sacheigentum dem Eigentümer keine uneingeschränkte Herrschaft über die Sache gibt, sondern sein Inhalt und seine Schranken nach Artikel 14 des Grundgesetzes durch das Gesetz festgelegt werden. ${ }^{215}$

Für die Reformer von 1965 waren Eigentum und geistiges Eigentum also noch grundlegend unterschiedlich. Die dafür verwendeten Argumente gelten heute zweifellos weiter. Für die folgende Argumentation zum Unterschied von Sacheigentum und immateriellem Eigentum wird auf (Kreutzer 2008, S. 133 ff) zurückgegriffen, der sich ausführlich kritisch mit der oft vorgenommenen Gleichbehandlung von geistigem und Sacheigentum auseinandersetzt. Seine Argumente werden hier auf das Wissenschaftsurheberrecht bezogen:

(1) Geistiges Eigentum wird, was die vermögensrechtlichen Interessen betrifft, ohnehin - anders als das Sacheigentum - nur für einen begrenzten Zeitraum geschützt (derzeit 70 Jahre nach dem Tod des Urhebers).

(2) Immaterielle Werke haben eine weitaus höhere Bedeutung ,für den kulturellen und geistigen Fortschritt“" als Objekte des Sacheigentums.

(3) Zudem wirken, so Kreutzer, „Beschränkungen der ausschließlichen Verfügungsgewalt des geistigen Eigentümers im Allgemeinen nicht so schwerwiegend wie zumeist Beschränkungen des Sacheigentums." ${ }^{\text {"216 }}$

(4) Wissenschaftliche Urheber, ganz in Übereinstimmung mit der hier vertretenen Symmetrie-These (Einheit von Urheber und Nutzer), haben selbst kein Interesse an einem Schutz, der Andere ausschließt. Kreutzer: „Baut der Urheber auf den Leistungen anderer auf, müssen auch diese wiederum von seinem Schaffen profitieren und diese nutzen können“. Bedient sich der Urheber aus dem verfügbaren Allgemeingut, ,ist es angemessen, dass auch er wieder etwas zurückführen muss.“

(5) Ein weiterer Unterschied besteht darin (s. das obige Zitat aus der 1965er Begründung), dass immaterielle Produkte, so Kreutzer, „dazu bestimmt sind, anderen zugänglich zu sein. Das Zugangsrecht dient hier im Gegensatz zum

215 Abschnitt 6 der Begründung für Schranken des Urheberrechts in der UrhR-Reform von 1965: https://bit.ly/2QRoIEg.

216 Vgl. (Rose 2003) ... Traditions of public property in the information age. Sie unterscheidet zwischen "Intellectual Space” (IS) und “Tangible Space”: „exclusive property rights come up long in Tangible Space but rather short in Intellectual Space“; zudem können IS-Objekte nicht durch Übernutzung vernichtet werden. 
Sacheigentum nicht in erster Linie der Möglichkeit, andere auszuschließen, sondern dem Rechtsinhaber die Entscheidung über den Zugang zum Werk und dessen Modalitäten vorzubehalten.“

(6) Sacheigentum ist immer an einen bestimmten Gegenstand an einem bestimmten Ort gebunden. „Dagegen ist das [immaterielle] Werk reproduzierbar und ubiquitär.“

Immaterielle Werke sind auch im Sinne der ökonomischen Gutstheorie gemeinschaftliche Güter (common goods), die grundsätzlich durch die Eigenschaften der Nicht-Ausschließbarkeit und der Nicht-Rivalität der Nutzung charakterisiert sind. Immaterielle Güter unterliegen anders als materielle Güter nicht dem Knappheitsprinzip und verbrauchen sich nicht im Gebrauch. Sie werden erst durch entsprechende Nutzungsrechte (wie durch das Urheberrecht) ökonomisch verwertbar. Die Unterschiede zwischen „Eigentum“ und „Geistigem Eigentum“ sind offensichtlich so gravierend, als dass das eine auf das andere übertragen werden kann (Kreutzer 2008):

\footnotetext{
Es zeigt sich also, dass zwischen geistigem und Sacheigentum derart elementare Unterschiede bestehen, dass die Interessenlage bei der einfach gesetzlichen Ausgestaltung beider Rechte nicht gleichgesetzt werden kann. Eine Übertragung der zum Sacheigentum entwickelten Grundsäte verbietet sich daher ebenso, wie eine Unterscheidung und Andersbehandlung auch vor dem Gleichheitsgrundsatz - generell unbedenklich erscheint. (a. a. O., 136)
}

Fazit. Der Gesetzgeber hat bis heute keinen Bedarf gesehen, „geistiges Eigentum“ als Benennung aufzugreifen - weder explizit durch eine Änderung im Grundgesetz oder an einer Stelle im UrhG. Eine explizite Erwähnung war wohl deshalb als überflüssig angesehen worden, weil diese Formulierung im internationalen völkerrechtlich verbindlichen Kontext (WIPO, TRIPS/WTO) üblich geworden ist und es auch in der EU-Charta heißt „Geistiges Eigentum wird geschützt“ (Art. 17 Abs. 2). Die Charta ist verbindlich für alle Mitgliedsländer. Das Konzept des geistigen Eigentums wird in der wissenschaftspolitischen Diskussion bis heute nicht in Frage gestellt schon gar nicht mit Blick auf Bildung und Wissenschaft. Die Argumente, die eindeutig gegen eine strikte Übertragung von „Eigentum“ auf „geistiges „Eigentum“ sprechen, werden bis heute vom Gesetzgeber nicht angenommen.

\subsection{Vermögensrechtliche Begründung des geistigen Eigentums durch das BVerfG}

Ist der Begriff, das Konzept des geistigen Eigentums im Urheberrecht seit langem präsent, so hat die Benennung „geistiges Eigentum“ in den Entscheidungen des 
BVerfG nach anfänglichem Zögern erst ab Mitte der 70er Jahre Eingang gefunden. In der Entscheidung „Schulbuchprivileg“ von 1971 wird „geistiges Eigentum” in Anführungszeichen verwendet (NR. 19). Das kann als „,sogenanntes geistiges Eigentum“ interpretiert werden. Sonst wird nur „Eigentum“ verwendet. In „Kirchenmusik“ von $1978^{217}$ wird der Begriff des geistigen Eigentums durch Übertragung von „Eigentum“ auf „geistiges Eigentum” als „feststehender Rechtsbegriff gehandhabt“ (Jänich 2002, 144) und ist seitdem als „geistiges Eigentum“ im Sprachgebrauch des BVerfG (und damit in der gesamten Fachwelt) fest verankert. Man kann davon ausgehen, dass auch das BVerfG in seinen hier einschlägigen Entscheidungen i. d. R. „geistiges Eigentum“ meint, auch wenn nur von „Eigentum“ gesprochen wird. In diesen (allerdings zeitlich weit zurückliegenden) Entscheidungen orientiert sich das BVerfG weniger an den (ansonsten als unstrittig angesehenen) Persönlichkeitsrechten und zieht auch das ideologische Leitbild des Urhebers als Schöpfer nicht besonders stark in die Begründung von Eigentum ein, sondern stellt die vermögensrechtlichen Gesichtspunkte und damit die Vergütungsaspekte in den Vordergrund. Das Urheberrecht, so ist es aus den Entscheidungen des BVerfG abzuleiten, solle die Schutzansprüche am Eigentum sehr stark über die vermögensrechtlichen Aspekte regeln:

Zu den konstituierenden Merkmalen des Urheberrechts als Eigentum im Sinne der Verfassung gehört die grundsätzliche Zuordnung des vermögenswerten Ergebnisses der schöpferischen Leistung an den Urheber im Wege privatrechtlicher Normierung und seine Freiheit, in eigener Verantwortung darüber verfügen zu können. Das macht den grundgesetzlich geschützten Kern des Urheberrechts aus. (BVerfG E-Schulbuchprivileg)

Das Bundesverfassungsgericht hat in BVerfG E 31, 229 (238ff.) entschieden, daß das vom Urheber geschaffene Werk und die darin verkörperte geistige Leistung in vermögensrechtlicher Hinsicht Eigentum im Sinne des Art. 14 Abs. 1 Satz 1 GG ist. Aus seiner verfassungsrechtlichen Gewährleistung erwächst dem Urheber die Befugnis, dieses „geistige Eigentum“ wirtschaftlich zu nutzen. (BVerfG E-Kirchenmusik)

Der Gesetzgeber ist im Rahmen des Regelungsauftrags nach Art. 14 Abs. 1 Satz 2 GG grundsätzlich verpflichtet, das vermögenswerte Ergebnis der schöpferischen Leistung dem Urheber zuzuordnen und ihm die Freiheit einzuräumen, in eigener Verantwortung darüber verfügen zu können. Im einzelnen obliegt ihm jedoch die Aufgabe, bei der inhaltlichen Ausprägung des Urheberrechts sachgerechte Maßstäbe festzulegen, die eine der Natur und sozialen Bedeutung des Rechts entsprechende Nutzung und angemessene Verwertung sicherstellen (ebda.)

217 BvR 352/71. 
Dieser dem immateriellen Eigentum zuzurechnender Vergütungsanspruch ist allerdings ein durchaus neues Phänomen, verursacht durch die immer größer werdende Bedeutung der materiellen Werte immaterieller Produkte. In der 1965er Fassung des UrhG war z. B. in Art. 11 nur von den „geistigen und persönlichen Beziehungen zum Werk“ die Rede (ausführlicher in 6.5). Der Bezug zum Anspruch auf Vergütung wurde erst 2002 durch Satz 2 von $\S 11$ explizit hergestellt. Damit soll nicht gesagt sein, dass erst seitdem die juristische Diskussion um Vergütungsansprüche entstanden ist. Aber es doch bemerkenswert, dass erst 2002 sich der Gesetzgeber veranlasst gesehen hat, Vergütungsansprüche explizit im Text von $\S 11$ zu verankern. Die vermögensrechtlichen Ansprüche gehen zusammen mit der Wahrnehmung der Verwertungsrechte (im Sinne eines kommerziellen Verständnisses von Verwertung). Sie werden den Urhebern exklusiv zugerechnet, aber können auch nach Einschätzung des BVerfGG keinesfalls absolut in jeder Hinsicht wahrgenommen werden. Entsprechend heißt es auch in der BVerfG Schulbuchprivileg-Entscheidung:

Diese grundsätzliche Zuordnung der vermögenswerten Seite des Urheberrechts an den Urheber zur freien Verfügung bedeutet aber nicht, daß damit jede nur denkbare Verwertungsmöglichkeit verfassungsrechtlich gesichert sei. Die Institutsgarantie gewährleistet einen Grundbestand von Normen, der gegeben sein muß, um das Recht als „Privateigentum“ bezeichnen zu können. Im einzelnen ist es Sache des Gesetzgebers, im Rahmen der inhaltlichen Ausprägung des Urheberrechts nach Art. 14 Abs. 1 Satz 2 GG sachgerechte Maßstäbe festzulegen, die eine der Natur und der sozialen Bedeutung des Rechts entsprechende Nutzung und angemessene Verwertung sicherstellen.

Im BVerfGG - Bearbeiter-Urheberrechte wird das konkreter spezifiziert:

Die Verfassung verpflichtet den Gesetzgeber nicht, „,ewige“ Urheber- oder Leistungsschutzrechte einzuräumen. Aus der Eigentumsgarantie ergibt sich zwar das Gebot, das wirtschaftliche Ergebnis der wiederschaffenden künstlerischen Tätigkeit dem Interpreten grundsätzlich zuzuordnen; dies bedeutet aber nicht, daß ihm jede nur denkbare Verwertungsmöglichkeit unbeschränkt zugewiesen werden müsse. Es ist vielmehr Sache des Gesetzgebers, im Rahmen der ihm zustehenden Gestaltungsbefugnis eine der Natur des Rechts entsprechende Regelung zu treffen, die den Interessen aller Beteiligten Rechnung trägt.

In Anschluss daran schränkt das BVerfG das geistige Eigentumsrecht durch die Zeitbeschränkung weiter ein:

Hiernach sind die im Urheberrechtsgesetz geregelten Befugnisse ihrem Wesen nach Rechte auf Zeit; sowohl die geistig-schöpferische als auch die wiederschaffende Leistung sind darauf angelegt, nach einiger Zeit frei zugänglich zu werden. Die Einräumung einer Bestandsfrist soll demgegenüber den Berechtigten eine angemessene wirtschaftliche, rechtlich festgelegte Verwertung ihrer Leistung sicherstellen. Überdies würden unbegrenzte Leistungs- 
schutzrechte zu erheblichen praktischen Schwierigkeiten führen, da schon nach wenigen Erbgängen die für den Rechtsverkehr notwendige Klarheit über den Rechtsinhaber entfällt.

Fazit. Es hat eine Weile gedauert, aber seit den siebziger Jahren ist deutlich erkennbar, dass das BVerfG im Zusammenhang von urheberrechtlichen Themen auch dann „geistiges Eigentum“ meint, wenn nur „Eigentum“ erwähnt wird. Die kommunikative Funktion von geistigem Eigentum („Mitteilungsgut“) ist in der BVerfGE immer mehr in den Hintergrund gerückt. Auch die „moralischen“ persönlichkeitsrechtlichen Aspekte von Eigentum spielen in hier einschlägigen Entscheidungen des BVerfG nicht die zentrale Rolle. Dominierend sind für die BVerfGE die vermögensrechtlichen Aspekte des geistigen Eigentums. In der starken Orientierung an den vermögensrechtlichen Aspekten bleibt das monistische Konzept des Urheberechts erhalten. Die ideell/moralisch begründeten Persönlichkeitsrechte werden als Grundlage für die Verwertungsrechte angesehen. Grundsätzlich wird hier beides in Frage gestellt - das auf das Individuum bezogene Konzept des Schöpfers und der vermögensrechtliche Anspruch von Wissenschaftlern auf Vergütung, insbesondere, wenn sie in einem vergüteten Arbeitsverhältnis tätig sind. Berechtigt ist diese Infragestellung vor allem deshalb, weil der Verwertungsbegriff in Bildung und Wissenschaft ein grundsätzlich anderer ist als der auf die kommerzielle Verwertung abzielende. Versteht man tatsächlich die Verwertungsrechte als Veröffentlichungsrechte für die Zugänglichmachung der Wissensobjekte für die Öffentlichkeit, dann sollte die monistische Tradition in diesem neuen Verständnis neu gefasst werden. Damit würde die vermögensrechtliche Begründung des geistigen Eigentums hinfällig und damit auch die Berechtigung, den Begriff des geistigen Eigentums im Wissenschaftsurheberrecht insgesamt zu verwenden.

\subsection{Begründung des geistigen Eigentums durch die Fiktionen des Schöpfers und des Immaterialguts}

Die verfassungsrechtliche Ableitung von Eigentum/geistiges Eigentum wird ergänzt durch die starke Stellung, die das Urheberrecht dem kreativen individuellen Schöpfer von Werken zubilligt. Die Einbettung des geistigen Eigentums des Schöpfers in das Urheberrecht wird häufig durch Bezug auf das Locke'sche Verständnis von Eigentum begründet. Jeder Mensch hat das Recht auf Eigentum an seiner Person, und das, was er durch Kraft seiner Hände, durch Arbeit hergestellt hat, ist als „Arbeitseigentum“ als Teil seiner Person anzusehen. Durch Arbeit wird das, was die Natur bereitstellt, erweitert und zum persönlichen exklusiven Eigentum, so 
dass eine nicht erlaubte Nutzung Dritter ausgeschlossen werden kann. Das Ausschlussrecht wird allerdings nicht so exklusiv gesehen, wie es heute für Eigentum der Fall ist. ${ }^{218}$ Locke hat nicht von ,geistigem Eigentum“ gesprochen. Das Schaffen eines Werks durch intellektuelle Arbeit kann aber als etwas angesehen werden, was so noch nicht da war - nicht in der Natur, aber auch nicht in den Werken Anderer. Mit diesem Kriterium begnügt sich i. d. R. auch das Urheberrecht (vgl. 5.2). Das durch Arbeit Erzeugte wird so zum Eigentum erklärt. Was bei Locke „Eigentum an seiner Person“ heißt, ist vergleichbar mit der Bestimmung des Werks als Ausdruck der Persönlichkeit seines Schöpfers (vgl. 5.3).

Was im Urheberrecht „Werk“ heißt, wird seit der vor allem durch Kohler angestoßenen Debatte auch „Immaterialgut“ genannt, und das Urheberrecht entsprechend als Teil des Immaterialgüterrechts (zusammen mit Patentrecht, Markenschutzrecht etc.). Seitdem gilt, dass das materielle Objekt, das Werkstück, das „rohe Artefakt“, wie es (Peukert 2018) nennt, z. B. das konkrete Buch in seinen vielfachen Kopien, durch das Urheberrecht nicht geschützt ist bzw. nicht geschützt werden kann. Vielmehr gilt der Schutz der geistigen immateriellen Repräsentation in dem vom Schöpfer hervorgebrachten Masterexemplar, welches sich dann auch in dem einzelnen Werkstück widerspiegelt.

Fazit. Der umfassende Schutz des individuellen Schöpfers/Urhebers, der sich im Schutz dessen geistigen Eigentums rechtlich verfestigt hat, ist entsprechend den Argumenten in Kap. 6 kein Fundament für ein Recht, das den Umgang mit Wissen und Information für Bildung und Wissenschaft regelt. Wird der individuelle Schöpferbegriff und wird die Annahme, dass das Werk Ausdruck der Persönlichkeit des Schöpfers sei, in Frage gestellt, ergibt auch die Verbindung von individuellem Autor und individuellen Eigentumsansprüchen keinen Sinn. Zusammen mit der für Bildung und Wissenschaft unangemessenen Reklamation von vermögensrechtlichen Ansprüchen durch die Veröffentlichung eines Werks sollte im Wissenschaftsurheberrecht nicht länger mit Ansprüchen des geistigen Eigentums argumentiert werden. In den folgenden Abschnitten wird das weiter ausgeführt.

218 „Die Arbeit seines Körpers und das Werk seiner Hände sind, so können wir sagen, im eigentlichen Sinne sein Eigentum. Was immer er also dem Zustand entrückt, den die Natur vorgesehen und in dem sie es belassen hat, hat er mit seiner Arbeit gemischt und ihm etwas eigenes hinzugefügt. Er hat es somit zu seinem Eigentum gemacht. Da er es dem gemeinsamen Zustand, in den es die Natur gesetzt hat, entzogen hat, ist ihm durch seine Arbeit etwas hinzugefügt worden, was das gemeinsame Recht der anderen Menschen ausschließt. Denn da diese Arbeit das unbestreitbare Eigentum des Arbeiters ist, kann niemand außer ihm ein Recht auf etwas haben, was einmal mit seiner Arbeit verbunden ist. Zumindest nicht dort, wo genug und ebenso gutes den anderen gemeinsam verbleibt“ (TTG II 27, S. 216 f.) - zitiert bei E-Learning-Angebot „Rechtstheorie Online“ Dr. Tilmann Altwicker, Universität Zürich - https://bit.ly/2kahD4s. 


\subsection{Interessen am geistigen Eigentum}

Interessen am Schutz des geistigen Eigentums können bei verschiedenen urheberrechtlichen Akteursgruppen ausgemacht werden:

a) Interesse der Kunst-/Kulturschaffenden. Die öffentliche Diskussion über geistiges Eigentum wird überwiegend mit Blick auf Künstler oder andere Kulturschaffenden (in 2.4 mit Peifer „Sympathiefiguren“ des Urheberrechts genannt) außerhalb von Bildung und Wissenschaft geführt. Ob heute noch der immer wieder als romantisch bezeichnete Genie-Schöpferbegriff selbst für diese Akteursgruppen zutrifft bzw. diese sich so bestimmen, sei dahingestellt. Aber dass diese i. d. R. auf eigene Rechnung arbeitenden Personen einen Anspruch darauf haben, für die Nutzung ihrer erstellten Werke eine angemessene Vergütung zu erhalten, findet allgemeine Zustimmung und muss hier nicht weiter problematisiert, also nicht in Frage gestellt werden. Ob die Begründung der Rechte von diesen Akteuren über das Eigentumsprinzip erfolgen muss, ist allerdings nicht zwingend. Es reichte einfach „Exklusive Rechte an ihren Werken“.

b) Ökonomische Verwertungsinteressen. Verleger sind i. d. R. die striktesten Vertreter für den Schutz der über das geistige Eigentum abgeleiteten Rechte. Sie sind als auf Einnahmen angewiesene Unternehmer an der vermögensrechtlichen Realisierung durch Vergütung interessiert und haben auch den größten Anteil an den durch kommerzielle Verwertung erzielten Vergütungen. Niemand bestreitet, dass für die auf kommerzielle Verwertung abzielende Erstellung von Informationsobjekten/-produkten aus den von Urhebern erstellten Wissensobjekten/Werke eine Vergütung reklamiert werden darf. Es handelt sich dabei aber nicht um Originalprodukte - diese werden als Wissensobjekte von den Urhebern erstellt -, sondern um Sekundärprodukte. Sie dürfen diese erstellen, weil sie per Vertrag die den Urhebern zustehenden Verwertungsrechte als Nutzungsrechte erworben haben. Trotzdem tut das Urheberrecht so, als ob die Verwerter durch den Erwerb der Nutzungsrechte an den Werken der Urheber gleichsam Eigentümer der Werke geworden sind. Das ist aber eine Fiktion (ganz im Sinne von Vaihingers Als-ob-Philosophie) - von der Urheberrechts-Community allerdings eine weitgehend als Realität akzeptierte Fiktion. Müssen Vergütungsansprüche von Verlegern wirklich über ein dem Urheberrecht zugerechnetes Eigentumskonzept begründet werden? Muss der Anspruch auf Schutz der kommerziellen Verwertung der daraus erstellten Werke überhaupt über das Urheberrecht geregelt werden. Könnten dies nicht über entsprechende Rechtsverhältnisse im Sachenrecht (BGB) geregelt werden? 
c) Geistiges Eigentum - kein Konzept in der Wissenschaft. Die ökonomische Situation von Wissenschaftlern ist eine ganz andere als bei Kunstschaffenden. Abgesehen von freischaffenden, also ohne institutionelle Bindung tätigen Wissenschaftlern stellt die Öffentlichkeit (bzw. Arbeitgeber in der Wirtschaft) für die Akteure in Bildung und Wissenschaft die Rahmenbedingungen bereit, damit Werke und Ausbildungsleistungen überhaupt geschaffen werden können. ${ }^{219}$

Kein Autor will, dass Andere seine Arbeiten vorzeitig oder gegen seine Erlaubnis in irgendeiner Form öffentlich machen oder diese durch unautorisierte Änderungen verfälschen. Jeder Autor hat das Recht, seine erarbeiteten Wissensobjekte für die Veröffentlichung zu nutzen. Veröffentlichung ist die Voraussetzung für die Wahrnehmung dieser Objekte durch Dritte und für eine dadurch erwartete Reputationssteigerung. Veröffentlichung ist die primäre Verwertung in der Wissenschaft. Dieses Recht, seine erstellten Wissensobjekte über Informationsobjekte nutzen zu können, sichert das jetzige Urheberrecht dem Urheber auch zu, nämlich bestimmen zu können, wann, „ob und wie sein Werk zu veröffentlichen ist“ ( $\$ 12$ Abs. 1). Mehr muss vom Wissenschaftsurheberrecht in dieser Hinsicht nicht erwartet werden.

d) Interesse der Öffentlichkeit, der Allgemeinheit. Wissen bzw. die daraus abgeleiteten Informationsprodukte werden nicht nur nach Ablauf der im Urheberrecht vorgesehenen Schutzfrist zu gemeinfreien Allgemeingütern, sondern sind grundsätzlich, sobald sie einmal öffentlich zugänglich gemacht wurden, Teil des allgemeinen kulturellen Erbes, für das für jedermann der Zugriff und die Nutzung gesichert sein müssen. Das wurde auch schon in der oben zitierten Begründung für die 1965-er Urheberrechtsreform so gesehen:

Als ein allgemeiner Grundsatz kann gelten, dass der Urheber insbesondere dort im Interesse der Allgemeinheit freien Zugang zu seinen Werken gewähren muss, wo dies unmittelbar der Förderung der geistigen und kulturellen Werke dient, die ihrerseits Grundlage für sein Werkschaffen sind.220

219 Wissenschaftler in privaten Beschäftigungsverhältnissen, aber auch die in öffentlich finanzierten Einrichtungen wie Bundes- oder Landesanstalten werden ebenfalls als Urheber im Sinne des Urheberrechts anerkannt, auch wenn die entsprechenden Arbeiten nicht selbstbestimmt, sondern direkt beauftragt worden sind. Diese Wissenschaftler werden aber für ihre im Arbeitsverhältnisse erstellten Werke kaum die Kategorie des Eigentums reklamieren können. Das zeigt sich auch darin, dass sie für die Nutzung ihrer Werke keine direkte Vergütung, auch keine Beteiligung an den Ausschüttungen der Verwertungsgesellschaften erwarten können.

220 Motive, UFITA 45 (1965 II), S. 278 - zitiert nach (Kreutzer 2008) Das Modell des deutschen Urheberrechts und Regelungsalternativen, $134 \mathrm{f}$. 
Ebenso gibt es dazu verschiedene Entscheidungen des BVerfG, die das besondere Interesse der Öffentlichkeit hervorheben, z. B. in den Schulbuchprivileg-Entscheidung von 1971:221

Mit der Publikation steht das geschützte Werk nicht nur dem Einzelnen zur Verfügung, es tritt zugleich in den sozialen Raum und kann damit zu einem eigenständigen, das kulturelle und geistige Bild der Zeit mitbestimmenden Faktor werden. Daher hat die Allgemeinheit ein bedeutsames Interesse daran, daß die Jugend im Rahmen eines gegenwartsnahen Unterrichts mit dem Geistesschaffen vertraut gemacht wird. Das gleiche gilt für Teilnehmer entsprechender Unterrichtsveranstaltungen. Die Verwirklichung dieser sozialen Aufgabe wäre aber nicht gewährleistet, wenn der Urheber die Aufnahme seines Werkes in eine Sammlung beliebig verhindern könnte.

Bei der verfassungsrechtlichen Beurteilung dieser gesetzlichen Schranke ist davon auszugehen, daß der Gesetzgeber nicht nur die Individualbelange zu sichern hat, sondern ihm auch aufgetragen ist, den individuellen Berechtigungen und Befugnissen die im Interesse des Gemeinwohls erforderlichen Grenzen zu ziehen; er muß den Bereich des Einzelnen und die Belange der Allgemeinheit in einen gerechten Ausgleich bringen. Die Verfassungsmäßigkeit der angefochtenen Vorschrift hängt somit - abgesehen von der sonstigen Übereinstimmung mit dem Grundgesetz - davon ab, ob sie durch Gründe des Gemeinwohls gerechtfertigt ist.

Vom Zeitpunkt seiner Publikation an entwickelt jedes Druckwerk ein Eigenleben. Es bleibt nicht nur vermögenswertes Ergebnis verlegerischer Bemühungen, sondern wirkt in das Gesellschaftsleben hinein. Damit wird es zu einem eigenständigen, das kulturelle und geistige Geschehen seiner Zeit mitbestimmenden Faktor. (in der Pflichtexemplar-Entscheidung von 1981)

Es ist, losgelöst von privatrechtlicher Verfügbarkeit, geistiges und kulturelles Allgemeingut. Im Blick auf diese soziale Bedeutung stellt es ein legitimes Anliegen dar, die literarischen Erzeugnisse dem wissenschaftlich und kulturell Interessierten möglichst geschlossen zugänglich zu machen und künftigen Generationen einen umfassenden Eindruck vom geistigen Schaffen früherer Epochen zu vermitteln. ${ }^{222}$

\subsection{Einschränkung der exklusiven Eigentumsrechte}

Unter d) des letzten Abschnitts wurde das Interesse der Allgemeinheit an der offenen Nutzung von Wissensobjekten herausgearbeitet, und dieses wurde durch verschiedene BVerfGE bestätigt. „Offen/Open” bedeutet, dass jedermann freien Zugang zu Objekten der öffentlich finanzierten Wissenschaft haben kann (Open Science).

221 BVerfGE 31, 229 - Schulbuchprivileg.

222 BVerfGE 58, 137 (S. 148 f.) - 14. Juli 1981 mit Hinweis auf BVerfGE 31, 229 - Schulbuchprivileg. 
Daraus folgt zwangsläufig, dass die Entscheidung darüber nicht eine subjektiv individuelle sein kann, sondern dass die Öffentlichkeit Ansprüche anmelden und durchsetzen kann. Eigentumsrechte in der Wissenschaft - wenn überhaupt davon die Rede sein kann - können keine exklusiven sein. Auch wenn, so die Position hier, Eigentumsrechte realitätsgerechter als Nutzungsrechte bezeichnet werden, kann auch das nicht zu individuellen Exklusivansprüchen führen. Rechte an den Wissensobjekten müssen zwischen den Produzenten und den Nutzern bzw. der Allgemeinheit geteilt werden.

Dass eine Einschränkung der exklusiven Eigentums- und entsprechenden Verwertungsrechte möglich ist, kann beim Patentrecht gesehen werden, welches ebenfalls dem geistigen Eigentum bzw. dem Immaterialgüterrecht zugerechnet wird. 2002 wurde durch die Novellierung von $\S 42$ des Arbeitnehmererfindungsgesetzes (ArbnErfG) das bisherige sogenannte „Hochschullehrerprivileg“ abgeschafft. Entsprechend diesem Privileg hatten bis dahin nur die Hochschullehrer selber das Recht der Patentierung bzw. der wirtschaftlichen Verwertung des von ihnen erarbeiteten Wissens, nicht aber die Hochschulen, in denen diese Wissenschaftler arbeiten. Mit der Novellierung können nun die mit öffentlichen Mitteln finanzierten Hochschulen selber das an ihnen entstandene Wissen in jeder Hinsicht verwerten, unbeschadet einer Entschädigung (vorgesehen sind $30 \%$ der Einnahmen) bzw. eines festzulegenden Nebenverwertungsrechts des/r dabei beteiligten Hochschullehrer. Diese Regelung des ArbnErfG hat auch zur Folge, dass das positive Publikationsrecht, welches allgemein als elementar für Wissenschaftsfreiheit angesehen wird, für eine festgesetzte Frist ausgesetzt ist, bis sich die Hochschule entschieden hat, für die Erfindung ein Patent zu beantragen. Wenn eine Erfindung einmal veröffentlicht wurde, ist diese nicht mehr als neu zu bewerten und ist daher auch nicht mehr patentierfähig. Ein Wissenschaftler muss daher eine geplante Publikation, in der eine möglicherweise patentfähige Erfindung enthalten ist, seiner Dienststelle melden, damit diese vorsorglich eine mögliche Patentierung anmelden kann. Dass diese Intensivierung der Meldepflichten der Wissenschaftler bzw. die intensivierten Patentierungsaktivitäten und von und aus Hochschulen zu einer Reduzierung der Publikationstätigkeit geführt hat, ist nicht zu belegen. Gegen diese Änderung im ArbnErfG hatte es damals erhebliche Widerstände gegeben. So hatte sich z. B. der Hochschullehrerverband entschieden (aber erfolglos) gegen diese Neuregelung gewandt, weil dies als starker Eingriff des Staates in Wissenschaftsfreiheit angesehen wurde. ${ }^{223}$ Es ist aber offenbar nie unternommen worden, gegen die Änderung des ArbnErfG als Eingriff in das geistige Eigentum und in Wissenschaftsfreiheit allgemein vor dem BVerfG zu klagen. Dem hatte

223 Deutscher Hochschulverband 9/2015 - https://bit.ly/37YxLcg. 
sich schon der BGH entgegengestellt. Er hatte anlässlich der Abschaffung des Hochschullehrerprivilegs 2002 - mit Bezug auf eine BverfGE von $1973^{224}$ - deutlich gemacht, dass die

„Freiheit von Forschung und Lehre [...] es allerdings nicht [gebietet], dass der Hochschullehrer auch Inhaber der Verwertungsrechte an seinen Forschungsergebnissen zu sein oder zu bleiben hat [...]. Die wirtschaftliche Zuordnung von geistigen Leistungen des Hochschullehrers fällt in den Normbereich des Art. 14 Abs. 1 Satz 1 GG, nicht des Art. 5 Abs. 3 GG. „[...] Das Grundrecht der freien wissenschaftlichen Betätigung [müsse] soweit unangetastet[bleiben], wie das unter Berücksichtigung der anderen legitimen Aufgaben der Wissenschaftseinrichtungen und der Grundrechte der verschiedenen Beteiligten möglich ist“..225

$\mathrm{Zu}$ diesen Aufgaben, auf deren Einhaltung der Staat auch zu sorgen habe, gehört auch die „Mittelaufbringung der Hochschule [...]. Der Funktionsfähigkeit der Institutionen des Wissenschaftsbetriebs [komme auch] Verfassungsrang zu [...]. Die grundrechtlich garantierte Freiheit von Forschung und Lehre erfordert nicht, dass den Forschern an Hochschulen die unbeschränkte Rechtsinhaberschaft an ihren dienstlich gemachten Forschungsergebnissen eingeräumt werden müsste [...].“(ebda.)

Exkurs zur Wissenschaftsfreiheit: Es ist zwar nicht unproblematisch, die Einschränkungen des Rechts auf Patentierung von Erfindungen auf die Einschränkung des Rechts der Urheber an ihren Wissensobjekten zu übertragen. Aber problematisch ist es, das Konzept des geistigen Eigentums als Begründung für Wissenschaftsfreiheit zu verwenden. ${ }^{226}$ Wissenschaftsfreiheit ist durch Art. 5 Abs. 3 Satz 1 GG umfassend gewährleistet: „Kunst und Wissenschaft, Forschung und Lehre sind frei." Wissenschaft wird im Allgemeinen als gemeinsamer Oberbegriff für Forschung und Lehre verstanden. Die BVerfG -Hochschul-Entscheidung 35, 79 hat dies ausführlich begründet:

Das in Art. 5 Abs. 3 GG enthaltene Freiheitsrecht schützt als Abwehrrecht die wissenschaftliche Betätigung gegen staatliche Eingriffe und steht jedem zu, der wissenschaftlich tätig ist oder tätig werden will [...]. Dieser Freiraum des Wissenschaftlers ist grundsätzlich ebenso vorbehaltlos geschützt, wie die Freiheit künstlerischer Betätigung gewährleistet ist. In ihm herrscht absolute Freiheit gegenüber jedem Eingriff öffentlicher Gewalt. In diesen Freiheitsraum fallen vor allem die auf wissenschaftlicher Eigengesetzlichkeit beruhenden Prozesse, Verhaltensweisen und Entscheidungen bei dem Auffinden von Erkenntnissen, ihrer Deutung und Weitergabe. Jeder, der in Wissenschaft, Forschung und Lehre tätig ist, hat - vorbehaltlich der Treuepflicht gemäß Art. 5 Abs. 3 Satz 2 GG - ein Recht auf Abwehr jeder staatlichen

224 Vgl. nur BVerfGE 36, 280, 291 = GRUR 1974, 142.

225 Vgl. BGH, Beschluss vom 18.9.2007 -X ZR 167/05 zur Regelung der „positiven Publikationsfreiheit“ des Hochschullehrers in $\$ 42$ Nr. 1 ArbEG.

226 (Kuhlen 2012a) Was haben Eigentum und Wissenschaftsfreiheit mit dem Urheberrecht zu tun? 


\begin{abstract}
Einwirkung auf den Prozeß der Gewinnung und Vermittlung wissenschaftlicher Erkenntnisse. Damit sich Forschung und Lehre ungehindert an dem Bemühen um Wahrheit als „etwas noch nicht ganz Gefundenes und nie ganz Aufzufindendes“ (Wilhelm von Humboldt) ausrichten können, ist die Wissenschaft zu einem von staatlicher Fremdbestimmung freien Bereich persönlicher und autonomer Verantwortung des einzelnen Wissenschaftlers erklärt worden. Damit ist zugleich gesagt, daß Art. 5 Abs. 3 GG nicht eine bestimmte Auffassung von der Wissenschaft oder eine bestimmte Wissenschaftstheorie schützen will. Seine Freiheitsgarantie erstreckt sich vielmehr auf jede wissenschaftliche Tätigkeit, d. h. auf alles, was nach Inhalt und Form als ernsthafter planmäßiger Versuch zur Ermittlung der Wahrheit anzusehen ist. Dies folgt unmittelbar aus der prinzipiellen Unabgeschlossenheit jeglicher wissenschaftlichen Erkenntnis.
\end{abstract}

Nicht nur die forschende und lehrende Tätigkeit, sondern auch die vorbereitenden und unterstützenden Handlungen gehören zur Wissenschaftsfreiheit. Dazu gehört der freie Zugriff zum publizierten Wissen. Das wird zwar auch durch die in Art. 5 Abs. 1 Satz 1 angesprochene Informationsfreiheit gewährleistet: ,Jeder hat das Recht, ... sich aus allgemein zugänglichen Quellen ungehindert zu unterrichten“; aber die unbestimmten Rechtsbegriffe ,allgemein zugänglich“ und „ungehindert“ sind stark auslegungsbedürftig. Für Wissenschaft wird alleine das Wort „frei“ verwendet. Jeder Wissenschaftler und jeder Lehrende muss in der Lage sein, das publizierte Wissen in Erfahrung bringen und dann für seine Forschung und seine Lehre nutzen zu können. Wissenschaftsfreiheit ist ohne Informationsfreiheit nicht vorstellbar. Wissenschaftsfreiheit ist auch Freiheit der Nutzung von Informationsobjekten. Trotzdem ist der Gesetzgeber durch die Grundgesetzsystematik gehalten, Grundrechte wie Wissenschafts- und Informationsfreiheit nicht kontextlos in positiven Gesetzen zu garantieren, sondern sie im Zusammenhang mit anderen Grundrechten zu sehen. Niemand kann Informations- oder Wissenschaftsfreiheit kontextlos für sich reklamieren. Erst recht gilt das aber für „geistiges Eigentum“.

Der Zusammenhang zwischen Grundrechten und positiven Gesetzen wird uns hier immer wieder beschäftigen. Es ist dabei auch deutlich zu erkennen, dass dieser Zusammenhang nicht zeitlos stabil ist, sondern viel Interpretationsspielraum offenlässt. Kreative Hermeneutik, Auslegung der Formulierungen in den Grundrechten und der bestehenden Gesetze, ist für Rechtsprechung und Rechtssetzung ein Erfordernis, um dem Zeitgeist, den Veränderungen der Rahmenbedingungen in den zu regulierenden Bereichen Rechnung tragen zu können. Inwieweit diese Hermeneutik auch verwendet wird, ist immer wieder Anlass für Streit zwischen den Vertretern, die dem originalen Wortlaut der Gesetze und Verfassungen streng folgen wollen, und denjenigen, die sich zwar auch nicht gegen das bestehende Recht wenden, aber einen weiteren Spielraum der Auslegung und Weiterentwicklung des Rechts sehen.

Wir stellen hier allerdings die grundsätzliche Frage, ob das Grundrecht auf Wissenschaftsfreiheit - verstanden als Freiheit in der Bestimmung des Forschungs- 
gegenstands, in der Wahl der Methoden der Forschung, als Recht auf Zugriff zum veröffentlichten Wissen und als Recht zur Veröffentlichung der erstellten Wissensobjekte - tatsächlich in Beziehung zum Grundrecht auf Eigentum bzw. hier auf geistiges Eigentum gesetzt werden muss. Oder auf den Punkt gebracht: Es wird hier abgelehnt, dass mit Berufung auf geistiges Eigentum - zumal wenn diese Berufung von den kommerziellen Verwertern erfolgt - urheberrechtliche Regelungen vorgenommen werden, die den freien Zugang und die freie verwertende Nutzung zu/von öffentlich gemachtem Wissen einschränken.

Zudem bedeutet Wissenschaftsfreiheit nicht uneingeschränkter Anspruch der Urheber auf ihre erstellten Wissensobjekte. „Forschung ist ... kein Selbstzweck"227, so hat das auch 2008 die Bundesregierung formuliert. Wissenschaftsfreiheit impliziert nicht nur Rechte, sondern auch Verantwortung gegenüber der sie finanzierenden Gesellschaft. Auch Entscheidungen des BVerfG haben bestimmt, dass der Urheber von Wissensobjekten nicht Anspruch auf jede Verwertung und entsprechende Vergütung hat. Um aus der Entscheidung des BGH zur positiven Publikationsfreiheit noch einmal zu zitieren: „Die grundrechtlich garantierte Freiheit von Forschung und Lehre erfordert nicht, dass den Forschern an Hochschulen die unbeschränkte Rechtsinhaberschaft an ihren dienstlich gemachten Forschungsergebnissen eingeräumt werden müsste [...]."228 Mit dieser Begründung wird in 11.3.6 die Einführung eines institutionellen Verwertungsrechts vorgeschlagen dort am Beispiel des Zweitverwertungsrechts - nicht als Ersatz für die individuelle Verwertung/Veröffentlichung, sondern als (nicht abdingbare) institutionelle Ergänzung. Das muss nicht und sollte nicht - die in 7.4 zahlreich angeführten Belege von BVerfG-Entscheidungen interpretierend -als verfassungswidrig eingeschätzt werden. ${ }^{229}$

227 Aus der Begründung des RegE zum Zweitverwertungsrecht - https://bit.ly/2NouagE.

228 Vgl. BGH, Beschluss vom 18.9.2007 -X ZR 167/05 zur Regelung der „positiven Publikationsfreiheit" des Hochschullehrers in $§ 42$ Nr. 1 ArbEG.

229 Möglicherweise könnte durch die Einführung eines institutionellen Rechts im Urheberrecht auch die Vergütungsproblematik in den Kontext der Abgeltungstheorie gesehen werden, die besagt, dass mit dem Lohn/Gehalt die Einräumung der dem Urheber zustehenden Nutzungsrechte (als Realisierung der Verwertungsrechte) abgegolten sind, „wenn der Arbeitgeber die im Rahmen der Verpflichtungen des Arbeitnehmers geschaffenen Werke zu betrieblichen Zwecken nutzt“ (Wandtke/Bullinger 4. Aufl. 2014, § 43,134). Das könnte auch für die Erstellung von Unterrichtsmaterialien gelten, welche z. B. Hochschulen im Rahmen des Fernstudiums bzw. von E-Learning-Kursen allgemein einsetzen wollen. Bislang orientiert sich die Rechtsprechung aber eher an der sogenannten Trennungstheorie, die zwischen Lohn und urheberrechtlichem Vergütungsanspruch strikt unterscheidet (Wandtke/Bullinger 4. Aufl. 2014, \$ 43,140); Gehalt setze die urheberrechtlichen Nutzungsrechte nicht außer Kraft. 


\subsection{Kritisch gegenüber geistigem Eigentum}

In den vorangegangenen Abschnitten ist immer wieder herausgearbeitet worden, dass das Urheberrecht gut ohne Referenz auf geistiges Eigentum auskommen könnte und dass diese insbesondere in Bildung und Wissenschaft dysfunktional geworden ist, d. h. die Weiterentwicklung und die Nutzung von Wissen eher behindert als befördert. Das ist keine singuläre subjektive Einschätzung. Tatsächlich ist der dominierenden juristischen Praxis, bei Wissen bzw. bei den Wissen enthaltenden Objekten von geistigem Eigentum zu sprechen, in den letzten Jahren immer häufiger widersprochen worden. Thomas Hoeren lehnte bei einer Anhörung vor der EnqueteKommission „Internet und Digitale Gesellschaft“ des Bundestags (5. Mai 2010 bis 5. April 2013) am 5.10.2010 die Verwendung der Bezeichnung ,geistiges Eigentum“ als gefährlich grundsätzlich ab: „Geistiges Eigentum ist ein dummer Begriff, bitte verzichten Sie darauf. Kampfbegriff der Preußen für dumme Politiker, damit die das irgendwie als Eigentum verstehen. Ist gefährlicher Begriff, gehen viele unreflektiert ran. Besser: Immaterialgüterrecht. Applaus.“230 Ob „Immaterialgüterrecht“ tatsächlich die bessere Benennung ist, sei dahingestellt. Die oben durchgeführten Überlegungen zu der Kombination von „immateriell“ und „Gut“ sprechen dagegen.

Ebenso umfassend kritisch gegenüber „Geistigem Eigentum“ äußert sich Richard M. Stallman in seinem immer wieder zitierten Essay „Did You Say “Intellectual Property"? It's a Seductive Mirage”. ${ }^{231}$ Er greift vor allem die undifferenzierte Anwendung dieses Begriffs auf so unterschiedliche Sachverhalte wie Patentrecht, Markenrecht und Urheberrecht an: "Rejection of 'intellectual property' is not mere philosophical recreation. The term does real harm." Vor allem sei er schädlich und unangebracht mit Blick auf das Urheberrecht. Ebenso wurde im Zusammenhang der beiden World Summits on the Information Society (WSIS) von einigen zivilgesellschaftlichen Gruppen unternommen, den Exklusiv-/Monopolcharakter des geistigen Eigentums zu relativieren. So hatte die Arbeitsgruppe Patents, Copyright and Trademarks (PCT) Kompromisse zwischen Eigentumsmonopolen und dem Teilen von Wissen vorgeschlagen:

Striking a balance between limited information monopolies, on the one hand, and the use and sharing of knowledge, on the other, is essential to the Information Society.

(Es ist für die Informationsgesellschaft von enormer Bedeutung, einer Balance zwischen den Interessen der begrenzten geistigen Monopole einerseits und der freien Nutzung und Beteiligung am Wissen durch alle Menschen andererseits, zu finden. $)^{232}$

230 Dieses Zitat beruht auf einer Mitschrift von Markus Beckedahl (Mitglied der Enquete Kommission 2011) - https://bit.ly/2qQPZwh.

231 Richard M. Stallman o. J., Website GNU.org - https://bit.ly/2kzZEEx.

232 Georg C. F. Greve November 2003 - https://bit.ly/2kz4LoC. 
Tatsächlich hat die "Declaration of Principles - Building the Information Society: a global challenge in the new Millennium” von WSIS I I Genf 2003 dann unter No. 43 eine daran angenäherte Formulierung gefunden:

Intellectual Property protection is important to encourage innovation and creativity in the Information Society; similarly, the wide dissemination, diffusion, and sharing of knowledge is important to encourage innovation and creativity. Facilitating meaningful participation by all in intellectual property issues and knowledge sharing through full awareness and capacity building is a fundamental part of an inclusive Information Society.

Ob die starke Ausrichtung an den vermögenrechtlichen Aspekten von geistigem Eigentum wirklich den Interessen der Urheber entgegengekommen ist, kann bezweifelt werden. Die von Eckhard Höffner historisch ausführlich belegte These bezüglich der ökonomischen Auswirkungen der Regulierung des geistigen Eigentums besagt, dass „der Schutz des geistigen Eigentums ... nicht zu dem gewünschten Ergebnis [führte], sondern dem Markt ... praktisch jede Entwicklungsmöglichkeit geraubt“ hat (Höffner 2010, 255). Besser gestellt dadurch seien lediglich Bestseller-Autoren und die Rechteverwerter, alle anderen Beteiligten seien dadurch schlechter gestellt gewesen. ${ }^{233}$ Geistiges Eigentum für die primären Produzenten zu reklamieren hat, wie hier schon mehrfach herausgestellt, eher die ideologisch verschleiernde Funktion, kommerzielle Verwertungsinteressen durchzusetzen (vgl. 6.6) Auch Reto M. Hilty hat in einem Interview mit der SZ vom 31.3.2019 deutlich gemacht, dass der Bezug auf „geistiges Eigentum“ als Recht „einzelner Akteure“ problematisch sei:

Man muss mit dem Begriff „geistiges Eigentum“ sehr vorsichtig sein. Er suggeriert eine Gleichwertigkeit mit dem Sacheigentum. Es war aber nie Funktion des Urheberrechts oder auch des Patentrechts, einzelnen Akteuren ein bestimmtes Gut so zuzuweisen, wie etwa ein Produzent seine Maschinen als sein „Eigentum“ verteidigen kann. Es geht vielmehr um immaterielle Güter, die für die Allgemeinheit zum Teil von hoher Relevanz sind. ${ }^{234}$

\subsection{Fazit zum geistigen Eigentum}

Die Umsetzung (und damit Einklagbarkeit) des Grundrechts der Eigentumsgarantie in ein positives Recht, hier des Urheberrechts, ist hochkompliziert und zudem auch hochumstritten. Unumstritten ist, dass der durch das Schaffen eines immateriellen Werks entstandene Eigentumsanspruch, also der Schutz des dann so genannten geistigen Eigentums, nicht in jeder Hinsicht gewährleistet ist. Dafür sorgt das Grundgesetz. Auch ist unumstritten, dass es keinen umfassenden Anspruch für eine vermögensrechtliche Beanspruchung der Eigentumsgarantie gibt. Dafür hat

233 Vgl. dazu die Rezension von Sebastian Neurauter in: (2011) JIPITEC 60, para. 1.

234 Reto M. Hilty, Interview mit der SZ vom 31.3.2019 - https://bit.ly/2L19hqv. 
das BVerfG gesorgt. Damit aber ist es nicht getan. Im Wissenschaftsurheberrecht ist „Eigentum“ keine sinnvolle Kategorie ist - sowohl für die Sicherung der Rechte der originären Autoren als auch für die der sekundär operierenden Verleger. Ebenso wird diese Frage hier verneint, ob es einen unverzichtbaren Anspruch der Autoren in Bildung und Wissenschaft auf eine vermögenswirksame Anerkennung der Leistung geben muss (ausführlich dazu in Kap. 8).

Auf keinen Fall kann der Anspruch auf Rechte an geistigem Eigentum als unverrückbar und quasi naturgegeben reklamiert werden. Es bleibt dem Gesetzgeber überlassen, in welchem Ausmaß er Eigentumsansprüche gelten lassen will. Dafür hat auch das BVerfG immer wieder einen breiten Spielraum eröffnet. ${ }^{235}$ Grundrechte gelten nicht absolut bzw. können nicht in der wortwörtlichen Formulierung als rechtsverbindlich reklamiert werden. Sie müssen immer wieder aus dem Geist der Zeit vorurteilsfrei und kreativ interpretiert werden. Grundrechte können auch mit anderen Grundrechten kollidieren, wie z. B. mit Wissenschaftsfreiheit oder auch Informationsfreiheit im Allgemeinen. Dabei könnte es durchaus auch Aufgabe des Gesetzgebers sein, nicht nur Kompromisse zwischen den Ansprüchen verschiedener Grundrechte zu finden, sondern durchaus auch Prioritäten zu setzen. Mit Bezug auf das öffentliche Interesse, gemessen an der Förderung des Gemeinwohls, gibt es gute Gründe, Wissenschaftsfreiheit höher zu gewichten als die Eigentumsgarantie für private Interessen. ${ }^{236}$ Allerdings gibt es auch gute Gründe dafür, das individuelle Konzept der Wissenschaftsfreiheit nicht absolut zu setzen, sondern aus öffentlichem Interesse zwar nicht auszusetzen, aber doch ohne substantiellen Verlust einzuschränken. ${ }^{237}$

235 Vgl. z. B. die BVerfGE „Bearbeiter-Urheberrechte“ von 1971, die dem Gesetzgeber dafür einen breiten Spielraum für den Umgang mit Eigentum eröffnet: „Die Eigentumsgarantie und das konkrete Eigentum sollen keine unüberwindliche Schranke für die gesetzgebende Gewalt bilden, wenn Reformen sich als notwendig erweisen. Der Gesetzgeber ist bei einem Reformwerk nicht vor die Alternative gestellt, die nach dem bisherigen Recht begründeten subjektiven Rechte entweder zu belassen oder unter den Voraussetzungen des Art. 14 Abs. 3 GG zu enteignen; er kann individuelle Rechtspositionen umgestalten, ohne damit gegen die Eigentumsgarantie zu verstoßen.“ - https://bit.ly/2kxJwDu.

236 (Witschen 1998) Was verdient moralisch den Vorzug? führt dafür das ethische Prinzip der Präferenzregel ein: „Mit Hilfe einer Präferenzregel kann wenigstens ein maßgeblicher Grund erkannt werden, warum unter den in Rede stehenden Bedingungen einer bestimmten Handlung oder institutionellen Regelung den Vorzug verdient“ (S. 18).

237 Diese Diskussion wird später ausführlicher am Beispiel des Zweitverwertungsrechts geführt (11.3) Die Anbindung eines institutionellen Zweitverwertungsrechts an das dem Urheberrecht zustehenden Zweitverwertungsrecht stellt einen nicht unbilligen Einschnitt in die Wissenschaftsfreiheit dar - zumal das Erstveröffentlichungsrecht bzw. die negative und positive Publikationsfreiheit uneingeschränkt dem Urheber belassen bleibt. Mit dieser Präferenzregel (FN 236) könnte auch das in Unterabschnitt 11.3.6 behandelte Konstanzer Dilemma entschieden werden. 
Interessen am geistigen Eigentum sind (entsprechend $a-d$ in 7.4) vielfältig. Diese Vielfalt ist aber kein Beweis dafür, dass geistiges Eigentum zu den Fundamenten urheberrechtlicher Regulierung gerechnet werden sollte. Insbesondere für das Wissenschaftsurheberrecht kann darauf ohne Verlust verzichtet werden. Rechte an produzierten Wissensobjekten sind keine Eigentumsrechte, sondern Nutzungsrechte der Autoren, der Nutzer, der Öffentlichkeit und auch der Verwerter. Aus informationsethischer Sicht und der Einschätzung von Wissen als für jedermann frei nutzbare Commons ist das Interesse der Öffentlichkeit an einmal verfügbar gemachten Informationsobjekten als am höchsten zu bewerten. Viele Zitate aus BVerfGE belegen diese deutlich. Informationsobjekte sind Teil des „sozialen Raums“. Sie bestimmen das „kulturelle und geistige Bild der Zeit“. Dass das BVerfG einen „gerechten Ausgleich“ fordert, ist nicht unbillig - allerdings mit der Einschränkung, dass der „gerechte Ausgleich“ durchaus über gewichtete Prioritäten entstehen kann bzw. im Wissenschaftsurheberrecht entstehen sollte.

Die starke Verankerung von Eigentum als geistiges Eigentum gehört zu dem, was im Wissenschaftsurheberrecht nicht angemessen ist. Das Beharren auf Eigentumsansprüchen gehört zu den Als-ob-Phänomenen, zu den Fiktionen, die in der Gegenwart nicht mehr zu halten sind - weder aus der Sicht und den Interessen der Akteure in Bildung und Wissenschaft und erst recht nicht aus dem Interesse der Öffentlichkeit, Wissen nicht zu verknappen, sondern freizügig nutzen zu können. Es geht in Bildung und Wissenschaft nicht um die Sicherung von Eigentumsansprüchen, sondern, wie schon zu Beginn angedeutet, ganz konkret nur a) um Nutzungsrechte von Autoren, b) um Nutzungsrechte der Nutzer (i.d. R. identisch mit den Autoren), c) um Nutzungsrechte der Institutionen der Urheber und d) der allgemeinen Öffentlichkeit und e) um Nutzungsrechte kommerziellen Verwerter. 